\title{
Sequential onset and concurrent expression of miR-9 genomic loci in single cells contributes to the temporal increase of mature miR-9 in zebrafish neurogenesis
}

\author{
Soto, X. ${ }^{1}$, Minchington, T. ${ }^{1}$, Lea, R. ${ }^{1}$, Lee, J. ${ }^{2}$ and Papalopulu, ${ }^{1 *}$ \\ *correspondence: nancy.papalopulu@manchester.ac.uk
}

${ }^{1}$ Division of Developmental Biology and Medicine, School of Medical Sciences, Faculty of Biology Medicine and Health, The University of Manchester, Oxford Road, Manchester, M13 9PT, UK;

${ }^{2}$ Medicines Discovery Catapult, Block 35, Mereside, Alderley Park, Alderley Edge, Cheshire, SK10 4TG, UK 


\section{Abstract}

3 Gene expression oscillations of the Hes/Her family members of transcriptional

4 repressors are important for cell state transitions during neural development. The

5 input of miR-9 is necessary to constrain gene expression noise, allowing oscillations

6 to occur and to be decoded by downstream genes. Hes/Her dynamics are sensitive

7 to the amount of miR-9 present in the cell but the mechanism by which miR-9 is

8 quantitatively controlled is not known. In vertebrates, there are several distinct

9 genomic loci that produce the same mature miR-9, leading to a number of

10 possibilities of how the production of mature miR-9 may be regulated.

11 Here, we show that the expression of miR-9 increases spatially and temporarily over

12 zebrafish development. A detailed time course of the expression of 7 pri-miR-9

13 genomic loci shows that they have distinct temporal and spatial profiles, which may

14 be brought about by different numbers of $\mathrm{E} / \mathrm{N}$-boxes in their regulatory regions.

15 Focusing on pairs that are expressed in the same area of the hindbrain region,

16 namely pri-miR-9-1/pri-miR-9-4 and pri-miR-9-1/pri-miR-9-5, we find that they

17 sequentially activated during neurogenesis, concurrent with a known change in Her6

18 dynamics from noisy to oscillatory. Analysis of expression at the single-cell level

19 shows that although they are sequentially activated, early and late pri-miRs are

20 mostly concurrently transcriptionally active in the same cells. This finding supports

21 the idea that increased mature miR-9 is contributed, at least in part, by overlapping

22 activation of distinct loci. We propose that this may lead to an additive, sharp,

23 increase of mature miR-9 which in turn may lead to rapid changes in Her6 dynamics. 


\section{Introduction}

27 MicroRNAs are a class of small ( $\sim 22$ nucleotides) regulatory non-coding RNAs,

28 which regulate gene expression at the post-transcriptional level. These small RNAs

29 are processed from large microRNA primary transcripts (pri-miRs) into 70 90nt

30 precursors (pre-miRs) before further splicing into 22nt mature microRNA. miR-9 is

31 a highly conserved microRNA which is expressed predominantly in the central

32 nervous system (CNS) of vertebrates and plays a crucial role during CNS

33 development.

35 Previous work in xenopus, zebrafish and mice has shown that miR-9 is essential for

36 the correct timing of cell fate transitions during neurogenesis (Coolen et al., 2013)

37 (Bonev et al., 2011; Bonev et al., 2012). miR-9 post-transcriptionally targets many

38 transcription factors that are involved in neural development such as FoxG1 (Shibata

39 et al., 2008), Tlx (Zhao et al., 2009) and members of the Hes/Her helix-loop-helix

40 family of transcription factors, including Hes1 in mouse and Xenopus (Bonev et al.,

41 2011; Bonev et al., 2012) and Her6 in zebrafish (Coolen et al., 2012; Galant et al.,

42 2016; Soto et al., 2020).

44 The Hes/Her family of proteins are expressed dynamically in an oscillatory manner at

45 the ultradian timescale (Hirata et al., 2002; Shimojo et al., 2008). Hes/Her

46 oscillations are achieved by a negative feedback loop, whereby Hes/Her proteins

47 inhibit their own transcription coupled with a rapid turnover of protein and mRNA.

48 Instability of both protein and mRNA allows for levels of the protein to fall, de-

49 repression to occur and expression to resume, generating a cyclic pattern (Hirata et

50 al., 2002; Novak and Tyson, 2008). Indeed, both mRNA and protein of Hes family 
51 genes are unstable, for example, in mice, the half-life of Hes 1 mRNA is 24 minutes

52 and the HES1 protein half-life is in the order of 22 minutes (Hirata et al., 2002) and

53 the Her6 (Zebrafish orthologue) protein half-life is around 10 minutes (Soto et al.,

54 2020).

55

56 The instability of the mRNA, as well as the translation of the protein, are controlled

57 by microRNAs. Indeed, our previous work revealed that miR-9 regulation is important

58 for allowing the oscillatory pattern of expression of HES1 to emerge. However, the

59 amount of miR-9 is important as too much or too little miR-9 can lead to dampening

60 of HES1 oscillations (Bonev et al., 2012; Goodfellow et al., 2014). We have also

61 recently shown that in zebrafish, the dynamics of Her6 protein expression switch

62 from noisy to oscillatory and this coincides temporally with the onset of miR-9

63 expression in the hindbrain (Soto et al., 2020). Furthermore, when the influence of

64 miR-9 on her6 is removed experimentally, Her6 expression does not evolve away

65 from the "noisy" regime with a consequent reduction in progenitor differentiation. We

66 have interpreted this to mean that the miR-9 input is necessary to constrain gene

67 expression noise, enabling oscillations to occur and to be decoded by downstream

68 genes (Soto et al., 2020). Together these findings support that Hes/Her dynamics

69 are sensitive to the amount of mature miR-9 present in the cell, however, the

70 mechanism by which this is controlled is not known.

72 The question is complicated by the observation that vertebrates (and some

73 invertebrates) possess multiple copies of the miR-9 gene at distinct loci but all

74 capable of producing the same mature microRNA. For example, both human and

75 mouse contain 3 copies of miR-9 (Rodriguez-Otero et al., 2011; Shibata et al., 2011) 
76 and frogs have 4 (Walker and Harland, 2008). Due to an additional round of whole-

77 genome duplications in teleost fish (Amores et al., 1998; Jaillon et al., 2004),

78 zebrafish have 7 paralogues of miR-9 (miR-9-1 to miR-9-7) (Chen et al., 2005).

80 One possibility is that different genomic loci contribute to miR-9 regulation in a

81 qualitative way, that is due to differential temporal and spatial specificity of mature

82 miR-9 expression. Indeed, there is some limited evidence that these discrete copies

83 of miR-9 are expressed differentially during development both temporally and

84 spatially (Nepal et al., 2016; Tambalo et al., 2020). Another, and yet unexplored,

85 possibility is that transcription from different loci may serve to control miR-9

86 quantitatively, that is to increase the amount of miR-9 in the cell and perhaps do so

87 in a temporally controlled manner.

89 Here, we undertake a systematic study of pri-miR-9 expression in zebrafish that aims

90 to address the likelihood of these distinct scenarios, with special attention to the

91 possibility of a quantitative control mechanism. We show by in situ hybridization that

92 the amount of mature miR-9 increases over zebrafish development, spreading from

93 the forebrain to the hindbrain between 24 and 48hpf. A detailed time course of the

94 expression of all 7 pri-miR-9 paralogues shows that they are all transcriptionally

95 active, but exhibit subtle, yet distinct, temporal and spatial profiles which could be

96 contributed by differences in their regulatory regions, for example, correlating with

97 the number of E/N-boxes present in the proximal promotor of each pri-miR-9.

98 Focusing on 2 pairs of early and late expressed pri-miR-9s, (pri-miR-9-1 and pri-miR-

99 9-4, or pri-miR-9-5) by fluorescent in situ hybridization at single cell level, we find 100 that, surprisingly, in some cells early and late pri-miR-9s were concurrently 
101 transcriptionally active. Taken together, we find evidence for a qualitative mechanism

102 in the deployment of pri-miR-9s, as well as a previously un-appreciated quantitative

103 component, both of which may contribute to the decoding function of mature miR-9s.

\section{RESULTS}

\section{All pri-mir-9s are expressed but with differed temporal onset}

107 To investigate the expression of the mature miR-9 in zebrafish embryos, we first 108 performed a whole mount in situ hybridization (WM-ISH) for the mature miR-9 using 109 an LNA (locked nucleic acid) probe. Mature miR-9 was detected only in forebrain at

110 24hpf (Fig. 1a) while at 30hpf miR-9 was observed in the midbrain and in

111 rhombomere $1(\mathrm{r} 1)$ of the hindbrain $(\mathrm{hb})$, maintaining high expression in forebrain

112 (Fig. 1a, 30hpf-red head arrow). Later in development miR-9 expression was seen in

113 the posterior hindbrain (Fig. 1a, 34-36hpf-blue arrow). As development progressed,

114 increased levels of expression throughout the hindbrain were observed while 115 expression in the forebrain decreased (Fig. 1a, 48hpf, blue arrow: hindbrain, green 116 arrow: forebrain). These results show a temporally controlled antero-posterior wave 117 of miR-9 expression along the brain/hindbrain axis.

119 In zebrafish, the mature miR-9 can be produced from seven paralogous of miR-9.

120 The miR-9 paralogues occupy 7 unique loci across the genome (GRCz11; Genome

121 Reference Consortium Zebrafish Build 11) (Yates et al., 2020). With the exception of

122 miR-9-3 which is located upstream of a lincRNA, all miR-9 genes are intragenic, 123 overlapping annotations of lincRNAs or proteins(Yates et al., 2020) (Figure S1a,b).

124 Our in silico analysis of previously published RNA-seq data confirms the differential 125 temporal expression of six of the seven miR-9 paralogues host genes (White et al., 
126 2017). It is also clear that expression of miR-9 host genes coincides with a gradual

127 decline in the expression of Her/Hes family genes expression, consistent with the

128 idea that hes/her genes are major targets of miR-9 (Figure S1c) (Bonev et al., 129 2011).

131 Previous work has revealed that the 7 miR-9 zebrafish paralogues are expressed in 132 forebrain at early stages of neurogenesis while toward the end are also expressed in 133 hindbrain (Nepal et al., 2016). However, little is known about the period spanning the 134 peak of neurogenesis, when miR-9 controls downstream targets such as the 135 ultradian oscillator Her6. To characterize the expression in greater spatiotemporal 136 detail, particularly over the hindbrain area where Hes/Her target genes are 137 expressed, we investigated the nascent expression of all 7 precursors over a time 138 period spanning the peak of neurogenesis which occurs at 33hpf (Lyons et al., 2003) 139 using specific probes for each pri-miR-9 (Figure S2; Methods, Molecular cloning).

141 We observed that all pri-miR-9s were first expressed in the forebrain (24hpf), in a 142 regional specific manner, which was not further characterised here. At 48hpf they are 143 all also expressed in hindbrain (Fig. 1b,d, 24 and 48hpf and Figure S3a-c) 144 consistent with what has been described before (Nepal et al., 2016). Differential 145 expression was evident in the intermediate stages. Specifically, pri-miR-9-3, 9-4 and 146 9-5 were expressed ahead of the others in the hindbrain. Here, pri-miR-9-4 was 147 evenly and highly expressed while pri-miR-9-3 and pri-miR-9-5 were expressed in a 148 gradient from anterior to posterior (Fig. 1b,c; 30hpf; blue arrowhead). At the peak of 149 hindbrain neurogenesis (34-36hpf), pri-miR-9-2 and pri-miR-9-7 were upregulated, 150 joining most of the pri-miR-9s that were highly expressed at this stage (Fig. 1b, 34- 
151 36hpf). Pri-miR-9-1 and miR-9-6 were temporally delayed until 48hpf, at which point

152 all pri-miR-9 were fully expressed.

153 Thus, overall, every pri-miR-9 was expressed in the CNS and exhibited similar but

154 unique expression patterns and a temporal progression. Characterising the temporal

155 order of the onset of expression in intermediate stages was a first step in asking

156 whether the expression of different pri-miR-9s is distinct or additive in the hindbrain,

157 which we address below.

Partially overlapping spatial expression of early pri-miR-9s

We selected three different precursors based on the onset of their hindbrain

162 temporal expression during development, earliest or latest, and the phylogenetic 163 analysis of precursors sequences in clades/subgroups performed by Alwin Prem

164 Anan et al., 2018. Pre-miR-9-1 was selected as the latest to express in the hindbrain 165 belonging to clade I/subgroup II, pre-miR-9-5 as the earliest to express that belongs 166 to clade I/subgroup I and pre-miR-9-4 as the earliest, belonging to clade II (Alwin 167 Prem Anand et al., 2018) (Figure S3d).

169 Double WM-FISH for pri-miR-9-1 and pri-miR-9-4 performed on stage 32hpf 170 embryos revealed expression of pri-miR-9-4 along the antero-posterior hindbrain 171 axis, while pri-miR-9-1 expression was limited to the region of the anterior hindbrain 172 corresponding to rhombomere 1 (Fig. 2a, red arrows). The transversal view of 173 rhombomere 4 reveals expression of pri-miR-9-4 within the ventricular zone, while 174 pri-miR-9-1 was almost completely absent (Fig. 2d, f). These data indicate that pri175 miR-9-s are mainly expressed in the region where most of the dorsal progenitors, 
176 medial progenitors and ventral progenitors are found (Fig. 2f) (Lyons et al., 2003;

177 Tambalo et al., 2020). The double WM-FISH experiment was repeated with pri-miR-

178 9-1 and pri-miR-9-5. At 32hpf, pri-miR-9-5 is evenly distributed antero-posteriorly in

179 the hindbrain whilst pri-miR-9-1 was mostly expressed in rhombomere 1 as one

180 would expect due to the delayed onset of expression for pri-miR-9-1 (Fig. 2b, red

181 arrows). Fluorescent intensity quantification of the region of interest (Fig. 2a,b

182 merge, yellow rectangle) show variability in the levels of expression, pri-miR-9-4 and

183 pri-miR-9-5 are significantly high expressed $(\mathrm{P}<0.05$ and $\mathrm{P}<0.0001$, respectively) to

184 pri-miR-9-1 (Fig. 2c), supporting the hypothesis of pri-miR-9-4 and pri-miR-9-5 early

185 expression. Transversal imaging of pri-miR-9-1/pri-miR-9-5 revealed similar pattern

186 expression to pri-miR-9-1/pri-miR-9-4 analysis. Interestingly, subtle differences were

187 observed in pri-miR-9-4 which had a broader pattern of expression, extending further

188 in the dorsal progenitor area than pri-miR-9-5 indicating some differences in spatial

189 expression between pri-miR-9s of the same temporal profile (Fig. 2d-e, compare

190 middle panels, Fig. 2f).

\section{Progressive additive expression of pri-miR-9s}

193 In order to examine whether the late expression of pri-miR-1 is cumulative with pri-

194 miR-4 and pri-miR-5, or whether it is spatially distinct, we analysed expression at 195 later stages. Coronal and transversal views of pri-miR-9-1 double WM-FISH with pri-

196 miR-9-4 and pri-miR-9-5 revealed both overlapping and distinct expression of the

197 primary transcripts (Fig. 3a-d). Distinct expression was most clearly observed in

198 transversal views that show pri-miR-9-5 is more restricted in its expression to the

199 middle-ventral progenitor region of the ventricular zone, while pri-miR-9-1 was more

200 broadly expressed toward the dorsal progenitor region (Fig. 3d, e). In order for the 
201 overlapping expression to contribute to the total levels of mature miR-9 in a cell,

202 early and late pri-miR-9s would need to be expressed in the same cells, which was

203 examined next.

204

205

Total mature miR-9 is contributed by overlapping activation of distinct miR-9

206 Ioci

207 To investigate the existence of a spatiotemporal overlap in pri-miR-9 expression at 208 the single cell level, we performed double WM-FISH for pri-miR-9-1 with pri-miR-9-4 209 and pri-miR-9-5. Cell boundaries were visualized with the cell boundary marker

210 BODIPY-texas red (BP-TR). At 48hpf we observed cells that have only one miR-9

211 precursor present (Fig. 4c,d,g,h) from which, cells expressing only pri-miR-9-1 were

212 often found in the dorsal progenitor region of the ventricular zone (Fig. 4a,c,e,g)

213 while cells expressing only pri-miR-9-4 or pri-miR-9-5 and not pri-miR-9-1 were

214 mostly observed in the middle-ventral progenitor region of the ventricular zone (Fig.

215 4a,d,e,h) suggesting differential spatial expression. However, we also found many

216 cells co-expressing two pri-miR-9s (Fig. 4a,b,e,f), suggesting that different miR-9

217 paralogues are concurrently transcriptionally active in the same cells.

219 Diverse regulation of miR-9 promoters

221 To have a better understanding of the origin of these spatial and temporal expression differences, we focused on the transcriptional

223 activation/repression/regulation of the different miR-9 precursors. Transcriptional

224 repressors such as Hes/Her family can inhibit expression through the binding to N/E-

225 boxes (Akazawa et al., 1992; Sasai et al., 1992). An E-box (enhancer box) is a DNA 
226 response element that can act as a protein-binding site of an activator or repressor,

227 while an N-box is a protein-binding for a repressor such as Hes1(Sasai et al., 1992).

229 We first performed an in-silico analysis to identify putative canonical DNA binding 230 motif of the bHLH transcription factors (E-boxes), with consensus sequence 231 CANNTG, in a $2 \mathrm{~kb}$ region upstream and $1 \mathrm{~kb}$ region downstream of the 232 transcriptional start site of the transcripts of the miR-9 paralogues as previously 233 defined in Chirag et al. 2016. We found miR-9-2 and miR-9-4 promotor/proximal 234 enhancer region contained the highest number of putative E-boxes, 21 and 18, 235 respectively, followed by miR-9-3, 5, 7, 1 and 6 (Fig. 5a, b). In concordance, the 236 bHLH transcription factor Neurogenin1 (Ngn1) is a pro-neural gene known to induce 237 miR-9 thus is a potential candidate to promote differential temporal expression of the 238 seven miR-9 paralogues through E-box binding (Zhao et al., 2015)

240 We next performed in silico analysis of the DNA binding motif sequence CACNAG

241 called, N-box. As previously mentioned, the transcription of miR-9 precursors is 242 repressed by Hes1 protein through this binding site (Bonev et al., 2012; Sasai et al., 243 1992). We identified variability within host gene regulatory sequences; 9 putative $\mathrm{N}$ 244 boxes were found in miR-9-5, $5 \mathrm{~N}$-boxes in miR-9-2, $3 \mathrm{~N}$-boxes in miR-6 while only 2451 in miR-9-7, in miR-9-4 and miR-9-1 and none in miR-9-3 (Fig. 5a, b). These results 246 may reflect variability of Hes1 strength regulation over the miR-9s proximal promotor 247 dependent on the number of $\mathrm{N}$-boxes that each miR-9 promoter/proximal enhancer 248 region contains. Indeed, we observed that expression levels of pri-miR-9-5 is 249 consistently reduced (Fig. 5e. dashed line) in the regions where the zebrafish Hes1 250 orthologues, Her6/Her9, are highly expressed (Fig. 5c. her6/her9 merge-white 
251 underlined), corresponding to the medial-dorsal progenitor region of the ventricular

252 zone and pri-miR-9-1 is expressed more broadly, with expression in the dorsal

253 progenitor region of the ventricular zone. We also observed expression of pri-miR-9-

2544 narrowed to the ventral progenitor region of the ventricular zone (Fig. $\mathbf{5 d}$, dashed

255 line), this can be explained by its higher number of E-boxes when compared to pri-

256 miR-9-1. Combined, these data outline the dynamical spatial expression of the highly

257 conserved microRNA, miR-9, based on the ability of repressors/activators to

258 modulate the different promoters of the seven different mir-9 precursors.

260 Discussion

261 miR-9 is expressed from several genomic loci which, after transcription and 262 processing, produce the same 5' mature form of miR-9 which is particularly 263 interesting because it targets the key neural progenitors Her/Hes TFs. How common 264 is this multi-locus organisation? In humans $6.3 \%$ of mature microRNA arms are 265 identical across two or more loci (Kozomara et al., 2019), thus, it is not very 266 common, but it is not unique to miR-9. In zebrafish this number rises to around $26732.3 \%$ (Kozomara et al., 2019). The higher number of microRNA expressed from 268 multiple loci is possibly due to the teleost-specific whole-genome duplication (WGD).

269 Evidence from rainbow trout also shows that following the salmonid-specific extra 270 round of WGD, microRNAs appear to be retained at higher levels than protein271 coding genes (Berhelot et al 2014). This may suggest that extra copies of microRNA 272 are evolutionary advantageous but why this is the case was not understood. Here, 273 we propose that retention of multiple microRNA loci could have specific functional 274 advantages for regulatory control of target gene expression of an organism. 
275 By examining in detail the temporal and spatial expression at single cell level of 3

276 select early and late pri-miR-9s, we offer two possible explanations for this multi-site

277 organisation or primary transcripts.

279 The first explanation involves a qualitative mechanism. In this scenario, distinct pri-

280 miR-9s have different spatial expression, which allows them to target different, i.e.

281 region-specific, genes. Some of the differences in the spatial expression of pri-miR-

282 9s are easily discernible macroscopically (e.g. differential expression in the

283 forebrain) while others are subtle and require post-hybridisation sectioning to

284 document, as we have done here. An example of the latter is the expression of pri-

285 miR-1 which extents more dorsally in the hindbrain than pri-miR-4. This correlates

286 well with the expression of her6 and her9, which are both miR-9 targets but are

287 expressed adjacent to each other along the D-V axis (Soto et al. 2020).

289 The second explanation favours a quantitative mechanism. In this scenario, the

290 differential temporal expression, where some primary transcripts commence their

291 expression early while others are only expressed late, results in the simultaneous

292 expression of both (or more) transcriptional loci in the same cells at a particular time

293 in development. In support of this scenario, we have shown by FISH that pri-miR-9-

294 1, a late onset pri-miR-9, is co-expressed in the same cells as the earlier onset pri-

295 miR-9-4 or -5 . Interestingly, we have not seen evidence for a mechanism where one

296 pri-miR-9 switches off and another one comes on, arguing against a mechanism

297 where an early pri-miR-9 "passes on" the task of repression to a later expressed one;

298 rather all loci seem to remain active at least for the duration of our observations

299 which covers the period of embryonic neurogenesis. This means that both early and 
300 late pri-miR-9s are concurrently transcribed at late stages, and assuming they are

301 both processed the amount of mature miR-9 in a cell could sharply increase. Given

302 that transcription saturates easily in many systems (Hafner et al., 2020), this co-

303 expression may be a strategy to increase the amount of miR-9 available to the cell

304 than what is possible with transcription from one locus alone. Indeed, we have

305 shown that the dynamical regime of Hes1 ( i.e. oscillatory expression to stable

306 expression at different levels) as well as the amount of time that Hes1 oscillates for,

307 depends on the amount of miR-9 in the cell (Bonev et al., 2012; Goodfellow et al.,

308 2014). More recently, we have shown by in vivo manipulations, that the input of miR-

3099 changes the dynamic expression of her6 from noisy to oscillatory (Soto et al., 310 2020).

312 Like many other miRs, miR-9 has been shown to be quite stable in xenopus and was

313 thus presumed to accumulate gradually over time (Bonev et al., 2012; Goodfellow et

314 al., 2014). In turn, changing the levels of miR-9 in the cell would drive the dynamics

315 of Hes 1 from one dynamical regime to another, as described above. While more

316 recent studies have questioned the long stability of miRs by using different methods

317 and showing that the stability of miR-9 varies between tissues (Kim et al., 2020;

318 Marzi et al., 2016; Ruegger and Grosshans, 2012), the onset of additive transcription

319 from two loci is nevertheless likely to exert a sharp increase in the amount of miR-9

320 present in the cell. It is possible that some genes/networks do not respond to slow

321 increases of miR-9 (a form of adaptation observed in signalling pathways (Dessaud

322 et al., 2007)), and a sharp, non-linear, increase may be needed to push a dynamical

323 system into a new state associated with a cell fate change. In fact, non-linearity of 
324 reactions is a key feature of systems that can generate oscillatory gene expression

325 (Novak and Tyson, 2008).

327 The qualitative and quantitative mechanisms suggested above are not mutually

328 exclusive and may be combined and also take place at the same time. A common

329 element is that both mechanisms would rely on distinct control of expression of

330 different miR-9 loci. In support of this, we have found that characteristics of the

331 regulatory regions and the organisation of pri-miR-9 in their host transcriptional units

332 show some distinct features. Interestingly, some of the regulatory region differences

333 may also be quantitative rather than simply qualitative; Indeed, we have found a

334 difference in the number of repressive $\mathrm{N}$-boxes but also differences in the number of

335 activators E-boxes, which may correlate with the differences in the extent of medio-

336 lateral (ontogenetically ventral-dorsal) expression of some pri-miR-9 pairs. Another

337 common element is that in both scenarios, a sequential temporal order of activation

338 can be involved, and indeed, we have observed a temporal sequence of activation

339 for most pri-miR-9s, roughly starting anteriorly and spreading posteriorly. This

340 temporal order was also observed when considering the entire miR-9 family and the

341 Her gene targets which were expressed on the whole earlier and were

342 downregulated when pri-miR-9s were upregulated.

343

344 Where multiple paralogues of a microRNA are present, differential qualitative and

345 quantitative regulation may be a more common feature than is currently appreciated.

346 In this respect, it is interesting that a recent study found that miR-196 paralogues

347 show both unique and overlapping expression. Single KOs showed some

348 redundancy but importantly, they also showed unique phenotypes and combinatorial 
349 KOs showed better penetrance together with additional defects, suggesting an

350 additive role of miR-196 paralogues in establishing vertebral number (Wong et al., 351 2015).

353 In conclusion, by providing evidence for both a quantitative and qualitative 354 mechanism, we have made conceptual advances on the possible roles of organising 355 pri-miR-9s in several distinct genomic loci, which may have led to their evolutionary 356 conservation. In addition, we highlight here some practical benefits of our work for 357 the experimenter; once mature miR-9 has been produced, it is not possible to tell 358 which genomic locus it was transcribed from. Thus, with multiple such loci being 359 potentially involved, it is very difficult for the experimenter to select the correct one to 360 tag, mutagenize or otherwise manipulate by CRISPR/Cas9. Therefore, an added 361 benefit of our work is that the detailed characterisation we have described here will 362 enable the selection of the correct genomic locus for genetic manipulation of miR-9 363 production, depending on the precise research question.

\section{Acknowledgements}

367 We are grateful to Dr Laure Bally-Cuif for sharing plasmids. The authors would also 368 like to thank the Biological Services Facility, Bioimaging and Systems Microscopy 369 Facilities of the University of Manchester for technical support. This work was 370 supported by a Wellcome Trust Senior Research Fellowship to NP (090868/Z/09/Z).

371 The funders had no role in study design, data collection and analysis, decision to 372 publish, or preparation of the manuscript. 
376 Conceptualization, X.S. and N.P.; Methodology, X.S. and N.P; Software, T.M. and

377 X.S.; Validation, X.S., T.M and R. L.; Formal Analysis, X.S. and T. M. ; Investigation,

378 X.S., R.L, J. L. and T.M.; Resources, N.P. and X.S.; Data Curation, X.S. and T.M..;

379 Writing - Original Draft, X.S.; Writing - Review \& Editing, N.P., X.S. and T.M..;

380 Visualization, X.S., T.M and R.L.; Supervision, N.P. and X.S; Project Administration,

381 N.P and X.S.; Funding Acquisition, N.P.

382

Declaration of Interests

385 The authors declare no competing interests.

Methods

\section{Research Animals}

Animal experiments were performed under UK Home Office project licenses

(PFDA14F2D) within the conditions of the Animal (Scientific Procedures) Act 1986.

Animals were only handled by personal license holders.

\section{mRNA extraction and Quantitative real-time PCR (qRT-PCR)}

mRNA was extracted using Trizol from a pool of 10 zebrafish embryos. SuperScript

II (Invitrogen) was used to do reverse cDNA synthesis using hexamer primers. For

quantitative real-time PCR (qRT-PCR) cDNA samples were diluted to a

concentration of $50 \mathrm{ng} / \mu \mathrm{l}$ with $\mathrm{dH}_{2} \mathrm{O}$. Each qPCR reaction was prepared in triplicate in

398 a 96-well plate with each well consisting of $0.2 \mu \mathrm{M}$ each forward and reverse primer,

399 50ng cDNA and SYBR Green Mastermix (ThermoFisher). Zebrafish embryo

400 Reactions were run on Step One Plus Real-time PCR System (Applied Biosystems)

401 alongside negative controls. The data for each sample was normalized to the

402 expression level of $b$-actin and analysed by the $2^{-\Delta \Delta C t}$ method, normalized to miR-9-1

403 expression. For each primer pair, the PCR product was examined by gel 
404 electrophoresis and its melting curve to ensure a single fragment of the predicted

405 molecular weight.

406

407 Molecular cloning

408 RNA probes for pri-miR-9-1, pri-miR-9-2, pri-miR-9-4, pri-miR-9-5 and pri-miR-9-7

409 were PCR amplified and cloned into pCRII vector using primers described in Table

410 S1. Except for pri-miR-9-2 probe, they were designed to distinguish the primary

411 transcripts by including sequences, intron and exon, before and after each

412 microRNA processing, while also covering the sequence corresponding to mature

413 miR-9 (Figure S2). Since the mature miR-9 sequence is conserved between

414 paralogs, to avoid any cross-binding of probes to this sequence we mutated it on

415 each probe by using QuikChange II XL Site-Directed Mutagenesis assay. This

416 allowed us introduce deletions and single nucleotide exchange in specific regions of

417 the mature miR-9 sequence (Table S2; Figure S2; sequence highlighted in red).

418 pri-miR-9-3 and pri-miR-9-6 probes were generated from plasmids kindly gifted by

419 Laure Bally-Cuif (Nepal et al., 2016).

421 Whole mount chromogenic and fluorescence in situ hybridization and

422 sectioning

423 Chromogenic in situ hybridisation was performed as previously described by

424 Christine Thisse (Thisse and Thisse, 2008). Multicolour fluorescence in situ

425 hybridisation was modified from Hoppler and Vize (Lea et al., 2012) by developing

426 with tyramide amplification (Perkin Elmer) after addition of antisense RNA probes

427 and antibodies conjugated to horseradish peroxidase (Lea et al., 2012). 
428 Sections were obtained as described in Dubaissi (Dubaissi et al., 2012) with

429 modifications. Embryos were embedded in $25 \%$ fish gelatine and $30 \%$ sucrose for a

430 minimum of $24 \mathrm{hrs}$. $18 \mu \mathrm{m}$ thickness hindbrain sections were collected and

431 transferred onto superfrost glass slides. The slides were air dried overnight under the

432 fume hood and stained with $5 \mu \mathrm{M}$ BODYPI-TR (Thermo-Fisher Scientific) before

433 mounting with Prolong Diamond Antifade.

435 Imaging

436 Chromogenic in situs were imaged using a Leica M165FC with a DFC7000T camera.

437 Fluorescent in situ sections were imaged using Leica TCS SP5 upright confocal with

438 HCX PL APO CS 40.0x1.25 OIL UV lens or Olympus FLUOVIEW FV1000 confocal

439 with UPLSAPO 20X NA:0.75 lens or UPLSAPO 20X NA:0.75 lens.

441 Expression analysis of hes/her genes and microRNA hosts

442 For the in silico analysis of the microRNA host gene expression we downloaded the

443 time course RNA-seq data (TPM) from White et al. 2017 supplemental file 3. Here

444 we used the overlapping host genes as a proxy for the expression of the microRNA.

445 MicroRNA would not show up in standard RNA-seq analysis and there is no current

446 microRNA time course data. Host genes were identified as those with overlapping

447 annotations with the miR-9 genes. The host genes for each microRNA are in Table

448 S4. MiR-9-7 has no overlapping annotation at this time and is thus not reported on in

449 these data.

450 We filtered the RNA-seq data removing genes which were neither the host genes of

451 the microRNA or members of the Her family. 3 repeats for each stage of

452 development are included in the data and we averaged the expression across the 3 
453 repeats for each stage. The stages reported in the data are based on standard

454 embryonic stages in zebrafish development. However, we wanted to visualize the

455 expression in terms of hours and the stages were converted accordingly. Finally,

456 before plotting these data were z-scored to normalize the expression of each of the

457 genes so that we could compare changes in expression over time rather than

458 absolute levels. These data were then plotted using the heatmap.3 package in R.

459

460 N-box / E-box analysis

461 To assess the number of $\mathrm{N}$ and $\mathrm{E}$-box elements in the promoter/proximal enhancer 462 region of the transcriptional start site (TSS) of the microRNA host genes/pri-miRs 463 (Table S4) we downloaded the Danio rerio genome from Ensembl. Using the 464 genome coordinates from (Nepal et al., 2016) (supplementary Table-S4) for the miR-

4659 TSS we took a region of $2 \mathrm{~kb}$ upstream to $1 \mathrm{~kb}$ downstream of the TSS. The $\mathrm{N}$ and 466 E-boxes in each region were then counted. The $\mathrm{N}$ and E-box sequences can be 467 found in Table S5. These data were then plotted using R (R Core, 2019) and the 468 ggplot2 library (Wickham, 2016)

\section{$470 \quad$ Figure Legends}

471 Figure 1. Pri-miR-9 paralogues are expressed with different temporal onset. (a)

472 Representative example of chromogenic whole mount in situ hybridization (WM-ISH)

473 of miR-9 using miR-9 LNA 5'-Dig observed at different stages during development;

474 longitudinal view, anterior to the left. Green arrow: forebrain (fb) expression. Blue

475 arrow: hindbrain (hb) expression. (b) Chromogenic WM-ISH (Cro-WMISH) of

476 different pri-miR-9s using specific probes for each paralogue observed at different

477 stages during development; longitudinal view, anterior to the left. Blue arrowhead: 
478 expression in hb at 30-31hpf, light blue arrowhead: expression in hb at 34-36hpf,

479 blue arrow: expression in hb at 48hpf. (c) High magnification of hb region from

480 respective paralogue at 30-31 hpf. (d) Heatmap plot representing expression levels in

481 hindbrain; grade $0=$ no expression in hindbrain, grade $1=$ weak expression in

482 hindbrain (rhombomere 1 or 2 ), grade $2=$ distinct expression in $\mathrm{hb}$ and grade $3=$ 483 strong expression in hb.

485 Figure 2. Partially overlapping expression of pri-mIR-9s. (a, b) Representative 486 example of double Fluorescent WM-ISH (WM-FISH) labelling of pri-miR-9-1/pri-miR487 9-4 (a) and pri-miR-9-1/pri-miR-9-5 (b) in hindbrain, coronal view, from wild-type 488 embryo observed at 32hpf; merged images (a, b; bottom panel) indicate pri-miR-9489 4(a; magenta) and pri-miR-9-5 (b; magenta) are expressed along the antero490 posterior axis while pri-miR-9-1 is limited to anterior hindbrain (a, b; green, red 491 arrows). Annotation denotate hindbrain (HB). (c) Pixel intensity mean of pri-miR-9-1, 492 pri-miR-9-4 and pri-miR-9-5 observed in ROI (region of interest), delineated by the 493 yellow rectangle in (a) and (b). Error bar in Scatter dot plot represent mean and SEM 494 of 32-34hpf pri-miR-9-1 (28 slices, 6 embryos), 32-34hpf pri-miR-9-4 (8 slices, 3 495 embryos) and 32-34hpf pri-miR-9-5 (18 slices, 3 embryos). Kruskal-Wallis with 496 Dunn's multiple comparison test, significance: $p<0.05^{\star}, p<0.0001^{* \star * *}$. (d, e) 497 Representative example of double WM-FISH labelling of pri-miR-9-1/pri-miR-9-4 (d) 498 and pri-miR-9-1/pri-miR-9-5 (e) in hindbrain rhombomere 4 (r4), transversal view, 499 from wild-type embryo observed at 32hpf; merged images (d, e; right panel) indicate 500 pri-miR-9-4(d; magenta) and pri-miR-9-5 (e; magenta) are expressed in the 501 ventricular zone region while pri-miR-9-1 is almost absent (d, e; green). Scale bar 30 $502 \mu \mathrm{m}$. (f) Schematic representation of transverse section from 32hpf zebrafish 
503 hindbrain at the level of the otic vesicle. VZ: ventricular zone, region where most of

504 progenitor cells are located. MZ: mantle zone, region of ongoing neurogenesis.

505 Within the VZ there are dorsal progenitors (DP), medial progenitors (MP) and ventral

506 progenitors (VP).

507

508 Figure 3. Progressive additive expression of pri-miR-9s. (a, b) Representative

509 example of double WM-FISH labelling of pri-miR-9-1 (left panel) and pri-miR-9-4

510 (middle panel) in hindbrain from wild-type embryo observed at 48hpf, coronal view

511 (a) and transversal view (b). (c, d) Representative example of double WM-FISH

512 labelling of pri-miR-9-1 (left panel) and pri-miR-9-5 (middle panel) in hindbrain from

513 wild-type embryo observed at 48hpf, coronal view (c) and transversal view (d);

514 merged images in coronal view (a, c; right) shows overlapping expression of the

515 primary transcripts yet in transversal view (b, d; right) reveals distinct expression,

516 pri-miR-9-1 is expressed more broadly toward the dorsal progenitor region. Scale bar

$51730 \mu \mathrm{m}$. hb: hindbrain (e) Schematic representation of transverse section from 48hpf

518 zebrafish hindbrain at the level of the otic vesicle. VZ: ventricular zone, region where

519 most of progenitor cells are located. MZ: mantle zone/marginal zone, region of

520 ongoing neurogenesis and mature neurons. Within the VZ there are dorsal

521 progenitors (DP), medial progenitors (MP) and ventral progenitors (VP).

522

523 Figure 4. mature miR-9 produced by overlapping activation of distinct miR-9

524 Ioci. (a-d) Representative example of double WM-FISH labelling pri-miR-9-1 and pri-

525 miR-9-4 combined with cell boundary staining, BODIPY-texas red (BP-TR). (b)

526 Indicates cells expressing both, pri-miR-9-1 (green) and pri-miR-9-4 (magenta). (c)

527 Indicates cells expressing only pri-miR-9-1 (green). (d) Indicates cells expressing 
528 only pri-miR-9-4 (magenta). (e-h) Representative example of double WM-FISH

529 labelling pri-miR-9-1 and pri-miR-9-5 combined with BP-TR. (f) Indicates cells

530 expressing both, pri-miR-9-1 (green) and pri-miR-9-5 (magenta). (g) Indicates cells

531 expressing only pri-miR-9-1 (green). (h) Indicates cells expressing only pri-miR-9-5

532 (magenta). Images were acquired from hindbrain $\mathrm{r} 4$, transversal sections, from 48hpf

533 wild-type embryos. Scale bars $30 \mu \mathrm{m}$ and $5 \mu \mathrm{m}$.

534

535 Figure 5. Diverse regulation of miR-9 promoters. (a) Bar plot showing the number

536 of $\mathrm{N}$ (green) and $\mathrm{E}$ (blue) boxes around the TSS (2kb upstream, 1kb downstream) of

537 the miR-9 genes. (b) Scatter plot showing the relationship between the number of $\mathrm{N}$

538 boxes and $E$ boxes around the TSS (2kb upstream, $1 \mathrm{~kb}$ downstream) of the miR-9

539 genes. (c) Representative example of double WM-FISH labelling her9 (green) and

540 her6 (magenta) in hindbrain, transversal view, from wild-type embryo observed at

$54144 \mathrm{hpf}$, white line indicates dorsal and medial progenitor region. Scale bar $30 \mu \mathrm{m}$.

542 (d,e) Representative example of double WM-FISH labelling pri-miR-9-1 (green)/pri-

543 miR-9-4 (magenta) (d) and pri-miR-9-1 (green)/pri-miR-9-5 (magenta) (e) in

544 hindbrain, transversal view, from wild-type embryos observed at 48hpf. Dashed

545 white line indicates ventral progenitor region. Scale bar $30 \mu \mathrm{m}$.

547 REFERENCE

548 Akazawa, C., Sasai, Y., Nakanishi, S., and Kageyama, R. (1992). Molecular

549 characterization of a rat negative regulator with a basic helix-loop-helix structure

550 predominantly expressed in the developing nervous system. J Biol Chem 267, $551 \quad 21879-21885$. 
552 Alwin Prem Anand, A., Huber, C., Asnet Mary, J., Gallus, N., Leucht, C., Klafke, R.,

553 Hirt, B., and Wizenmann, A. (2018). Expression and function of microRNA-9 in the

554 mid-hindbrain area of embryonic chick. BMC Dev Biol 18, 3.

555 Amores, A., Force, A., Yan, Y.L., Joly, L., Amemiya, C., Fritz, A., Ho, R.K.,

556 Langeland, J., Prince, V., Wang, Y.L., et al. (1998). Zebrafish hox clusters and

557 vertebrate genome evolution. Science 282, 1711-1714.

558 Bonev, B., Pisco, A., and Papalopulu, N. (2011). MicroRNA-9 reveals regional

559 diversity of neural progenitors along the anterior-posterior axis. Dev Cell 20, 19-32.

560 Bonev, B., Stanley, P., and Papalopulu, N. (2012). MicroRNA-9 Modulates Hes1

561 ultradian oscillations by forming a double-negative feedback loop. Cell Rep 2, 10-18.

562 Chen, P.Y., Manninga, H., Slanchev, K., Chien, M., Russo, J.J., Ju, J., Sheridan, R.,

563 John, B., Marks, D.S., Gaidatzis, D., et al. (2005). The developmental miRNA

564 profiles of zebrafish as determined by small RNA cloning. Genes Dev 19, 1288-

5651293.

566 Coolen, M., Katz, S., and Bally-Cuif, L. (2013). miR-9: a versatile regulator of

567 neurogenesis. Frontiers in cellular neuroscience 7, 220.

568 Coolen, M., Thieffry, D., Drivenes, O., Becker, T.S., and Bally-Cuif, L. (2012). miR-9

569 controls the timing of neurogenesis through the direct inhibition of antagonistic

570 factors. Dev Cell 22, 1052-1064.

571 Dessaud, E., Yang, L.L., Hill, K., Cox, B., Ulloa, F., Ribeiro, A., Mynett, A., Novitch,

572 B.G., and Briscoe, J. (2007). Interpretation of the sonic hedgehog morphogen

573 gradient by a temporal adaptation mechanism. Nature 450, 717-720.

574 Dubaissi, E., Panagiotaki, N., Papalopulu, N., and Vize, P.D. (2012). Antibody

575 development and use in chromogenic and fluorescent immunostaining. Methods Mol

576 Biol 917, 411-429. 
577 Galant, S., Furlan, G., Coolen, M., Dirian, L., Foucher, I., and Bally-Cuif, L. (2016).

578 Embryonic origin and lineage hierarchies of the neural progenitor subtypes building

579 the zebrafish adult midbrain. Dev Biol 420, 120-135.

580 Goodfellow, M., Phillips, N.E., Manning, C., Galla, T., and Papalopulu, N. (2014).

581 microRNA input into a neural ultradian oscillator controls emergence and timing of

582 alternative cell states. Nature communications 5, 3399.

583 Hafner, A., Reyes, J., Stewart-Ornstein, J., Tsabar, M., Jambhekar, A., and Lahav,

584 G. (2020). Quantifying the Central Dogma in the p53 Pathway in Live Single Cells.

585 Cell Syst 10, 495-505 e494.

586 Hirata, H., Yoshiura, S., Ohtsuka, T., Bessho, Y., Harada, T., Yoshikawa, K., and

587 Kageyama, R. (2002). Oscillatory expression of the bHLH factor Hes1 regulated by a

588 negative feedback loop. Science 298, 840-843.

589 Jaillon, O., Aury, J.M., Brunet, F., Petit, J.L., Stange-Thomann, N., Mauceli, E.,

590 Bouneau, L., Fischer, C., Ozouf-Costaz, C., Bernot, A., et al. (2004). Genome

591 duplication in the teleost fish Tetraodon nigroviridis reveals the early vertebrate

592 proto-karyotype. Nature 431, 946-957.

593 Kim, C.K., Asimes, A., Zhang, M., Son, B.T., Kirk, J.A., and Pak, T.R. (2020).

594 Differential Stability of miR-9-5p and miR-9-3p in the Brain Is Determined by Their

595 Unique Cis- and Trans-Acting Elements. eNeuro 7.

596 Kozomara, A., Birgaoanu, M., and Griffiths-Jones, S. (2019). miRBase: from

597 microRNA sequences to function. Nucleic Acids Res 47, D155-D162.

598 Lea, R., Bonev, B., Dubaissi, E., Vize, P.D., and Papalopulu, N. (2012). Multicolor

599 fluorescent in situ mRNA hybridization (FISH) on whole mounts and sections.

600 Methods Mol Biol 917, 431-444. 
601 Lyons, D.A., Guy, A.T., and Clarke, J.D. (2003). Monitoring neural progenitor fate 602 through multiple rounds of division in an intact vertebrate brain. Development 130 , $603 \quad 3427-3436$.

604 Marzi, M.J., Ghini, F., Cerruti, B., de Pretis, S., Bonetti, P., Giacomelli, C., Gorski, 605 M.M., Kress, T., Pelizzola, M., Muller, H., et al. (2016). Degradation dynamics of 606 microRNAs revealed by a novel pulse-chase approach. Genome Res 26, 554-565.

607 Nepal, C., Coolen, M., Hadzhiev, Y., Cussigh, D., Mydel, P., Steen, V.M., Carninci, 608 P., Andersen, J.B., Bally-Cuif, L., Muller, F., et al. (2016). Transcriptional, post609 transcriptional and chromatin-associated regulation of pri-miRNAs, pre-miRNAs and 610 moRNAs. Nucleic Acids Res 44, 3070-3081.

611 Novak, B., and Tyson, J.J. (2008). Design principles of biochemical oscillators. 612 Nature reviews Molecular cell biology 9, 981-991.

613 R Core, T. (2019). R: A language and environment for statistical computing.

614 Rodriguez-Otero, P., Roman-Gomez, J., Vilas-Zornoza, A., Jose-Eneriz, E.S., 615 Martin-Palanco, V., Rifon, J., Torres, A., Calasanz, M.J., Agirre, X., and Prosper, F. 616 (2011). Deregulation of FGFR1 and CDK6 oncogenic pathways in acute 617 lymphoblastic leukaemia harbouring epigenetic modifications of the MIR9 family. Br J 618 Haematol 155, 73-83.

619 Ruegger, S., and Grosshans, H. (2012). MicroRNA turnover: when, how, and why. 620 Trends Biochem Sci 37, 436-446.

621 Sasai, Y., Kageyama, R., Tagawa, Y., Shigemoto, R., and Nakanishi, S. (1992). Two 622 mammalian helix-loop-helix factors structurally related to Drosophila hairy and 623 Enhancer of split. Genes Dev 6, 2620-2634. 
624 Shibata, M., Kurokawa, D., Nakao, H., Ohmura, T., and Aizawa, S. (2008).

625 MicroRNA-9 modulates Cajal-Retzius cell differentiation by suppressing Foxg1

626 expression in mouse medial pallium. J Neurosci 28, 10415-10421.

627 Shibata, M., Nakao, H., Kiyonari, H., Abe, T., and Aizawa, S. (2011). MicroRNA-9

628 regulates neurogenesis in mouse telencephalon by targeting multiple transcription

629 factors. J Neurosci 31, 3407-3422.

630 Shimojo, H., Ohtsuka, T., and Kageyama, R. (2008). Oscillations in notch signaling

631 regulate maintenance of neural progenitors. Neuron 58, 52-64.

632 Soto, X., Biga, V., Kursawe, J., Lea, R., Doostdar, P., Thomas, R., and Papalopulu,

633 N. (2020). Dynamic properties of noise and Her6 levels are optimized by miR-9,

634 allowing the decoding of the Her6 oscillator. EMBO J 39, e103558.

635 Tambalo, M., Mitter, R., and Wilkinson, D.G. (2020). A single cell transcriptome atlas 636 of the developing zebrafish hindbrain. Development 147.

637 Thisse, C., and Thisse, B. (2008). High-resolution in situ hybridization to whole-

638 mount zebrafish embryos. Nature protocols 3, 59-69.

639 Walker, J.C., and Harland, R.M. (2008). Expression of microRNAs during embryonic 640 development of Xenopus tropicalis. Gene Expr Patterns 8, 452-456.

641 White, R.J., Collins, J.E., Sealy, I.M., Wali, N., Dooley, C.M., Digby, Z., Stemple, 642 D.L., Murphy, D.N., Billis, K., Hourlier, T., et al. (2017). A high-resolution mRNA 643 expression time course of embryonic development in zebrafish. eLife 6.

644 Wickham, H. (2016). ggplot2, Elegant Graphics for Data Analysis (Springer 645 International Publishing).

646 Wong, S.F., Agarwal, V., Mansfield, J.H., Denans, N., Schwartz, M.G., Prosser, 647 H.M., Pourquie, O., Bartel, D.P., Tabin, C.J., and McGlinn, E. (2015). Independent 
648 regulation of vertebral number and vertebral identity by microRNA-196 paralogs.

649 Proc Natl Acad Sci U S A 112, E4884-4893.

650 Yates, A.D., Achuthan, P., Akanni, W., Allen, J., Allen, J., Alvarez-Jarreta, J.,

651 Amode, M.R., Armean, I.M., Azov, A.G., Bennett, R., et al. (2020). Ensembl 2020.

652 Nucleic Acids Res 48, D682-D688.

653 Zhao, C., Sun, G., Li, S., and Shi, Y. (2009). A feedback regulatory loop involving

654 microRNA-9 and nuclear receptor TLX in neural stem cell fate determination. Nat

655 Struct Mol Biol 16, 365-371.

656 Zhao, J., Lin, Q., Kim, K.J., Dardashti, F.D., Kim, J., He, F., and Sun, Y. (2015).

657 Ngn1 inhibits astrogliogenesis through induction of miR-9 during neuronal fate 658 specification. eLife 4, e06885. 
Figure 1

a

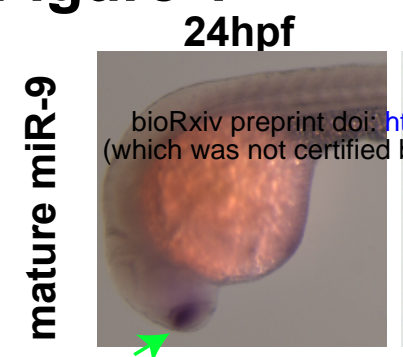

$30 \mathrm{hpf}$

35-38hpf

48hpf

miR-9 LNA 5'
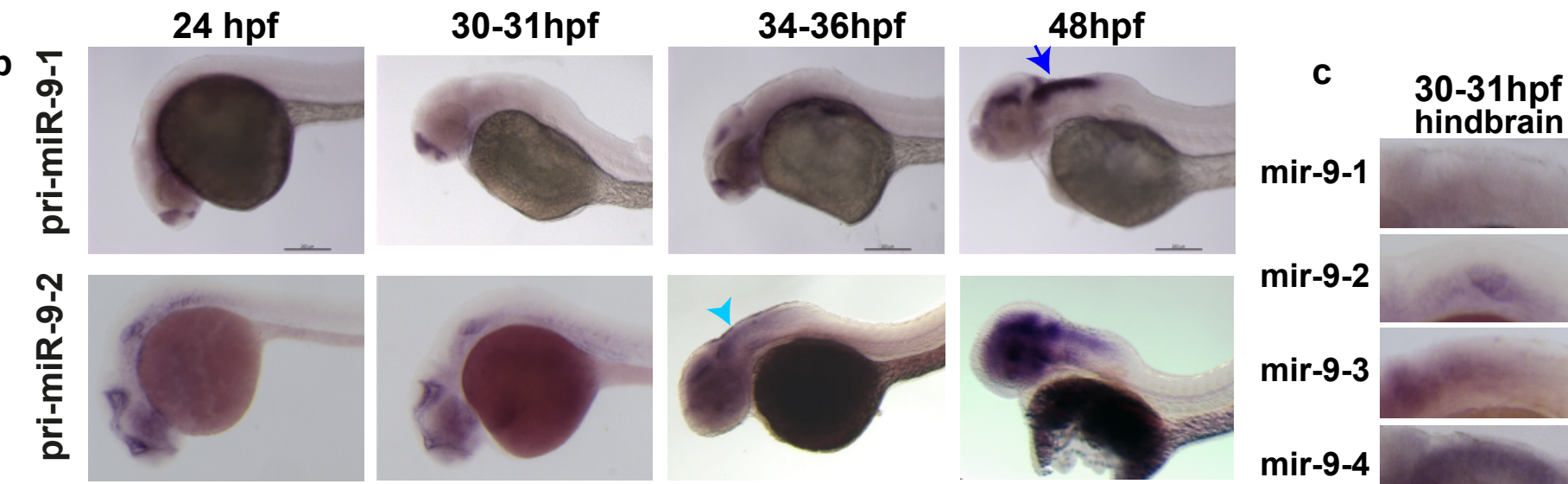

mir-9-1

hindbrain
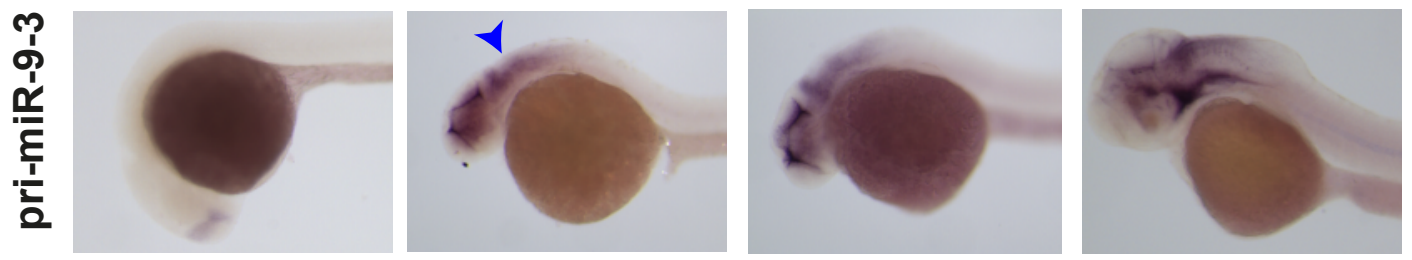

mir-9-2

mir-9-3

mir-9-4

mir-9-5

mir-9-6
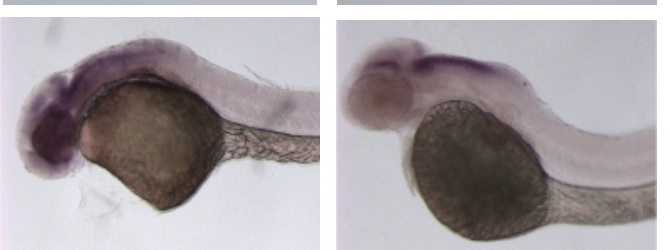

mir-9-7

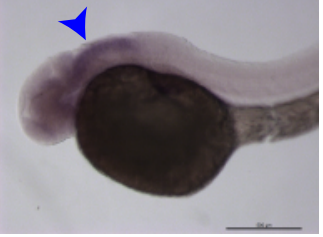

mir-9-7

d
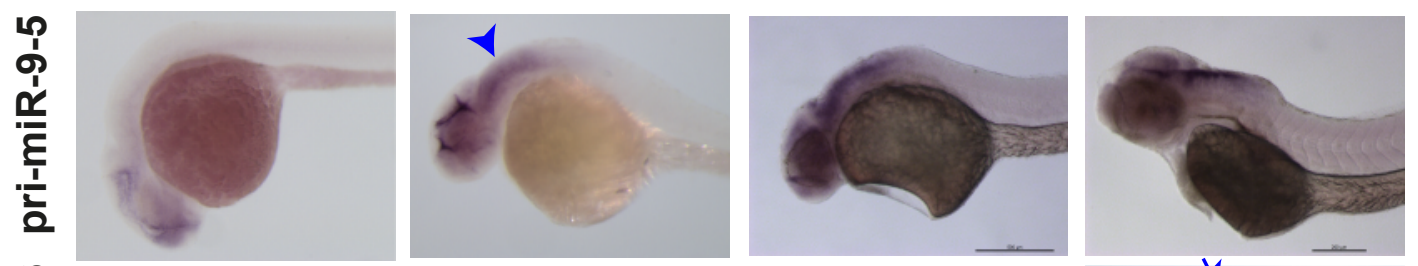

\begin{tabular}{l|l|l|l|l|} 
mir-9-1 & 0 & 0 & 1 & 3 \\
\cline { 2 - 5 } mir-9-2 & 0 & 1 & 2 & 3 \\
\cline { 2 - 5 } mir-9-3 & 0 & 2 & 2 & 3 \\
\cline { 2 - 5 } mir-9-4 & 0 & 3 & 3 & 3 \\
\cline { 2 - 5 } mir-9-5 & 0 & 2 & 3 & 3 \\
\cline { 2 - 5 } mir-9-6 & 0 & 1 & 1 & 3 \\
\cline { 2 - 5 } mir-9-7 & 0 & 1 & 2 & 3 \\
\cline { 2 - 5 } & & & \multicolumn{3}{|c}{}
\end{tabular}
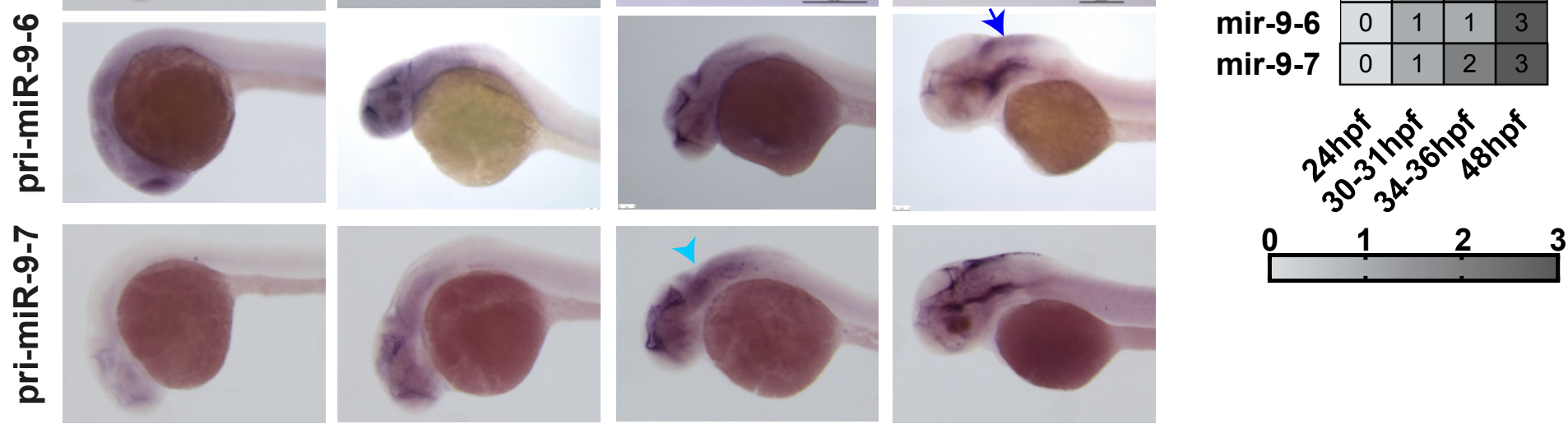


\section{Figure 2}

bioRxiv preprint doi: https://doi.org/10.1101/2020.08.03.233890; this version posted August 3, 2020. The copyright holder for this preprint (which was not certified by peer review) is the author/funder, who has granted bioRxiv a license to display the preprint in perpetuity. It is made available under aCC-BY 4.0 International license.

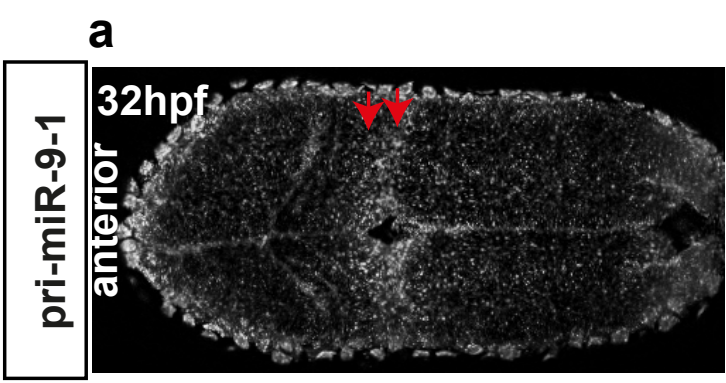

HB

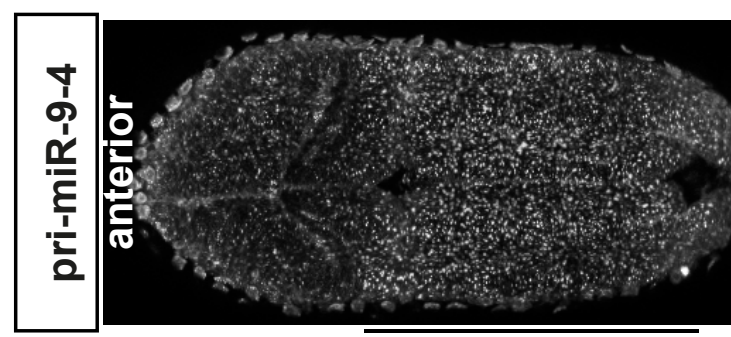

HB

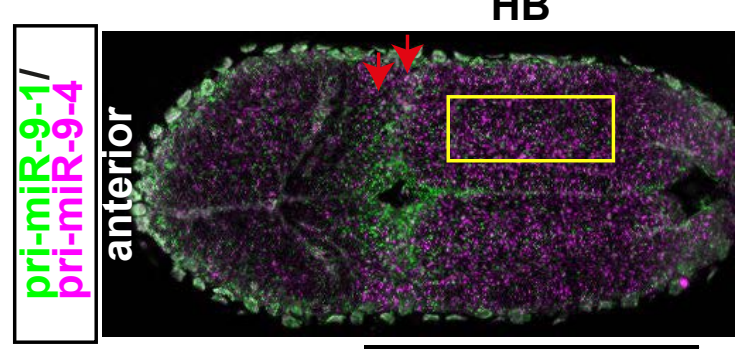

coronal view

d

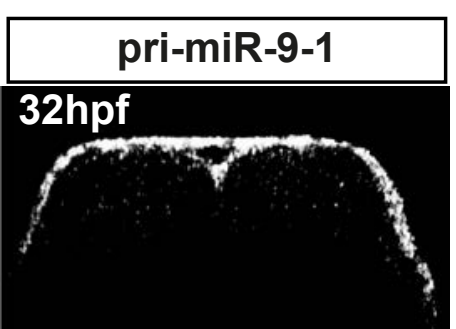

e

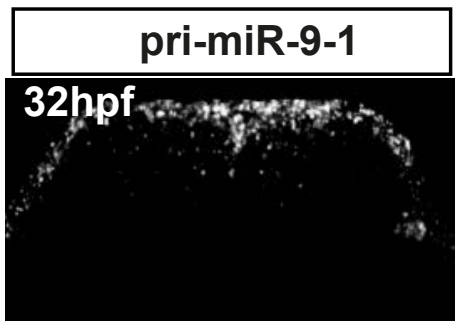

transversal view b

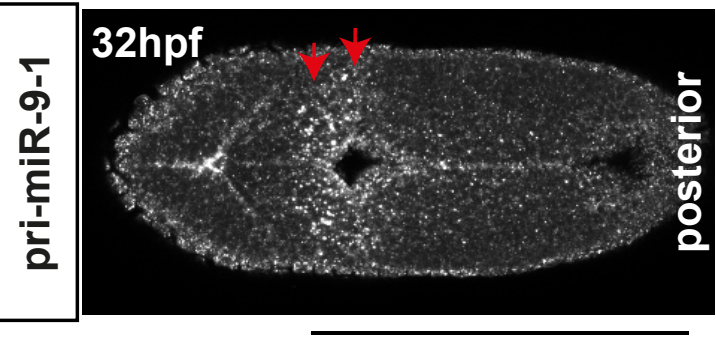

HB

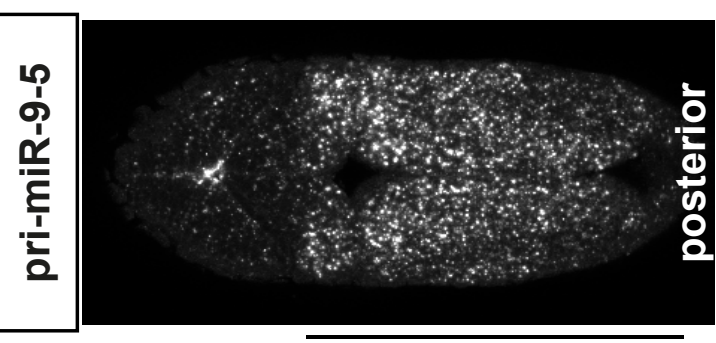

HB
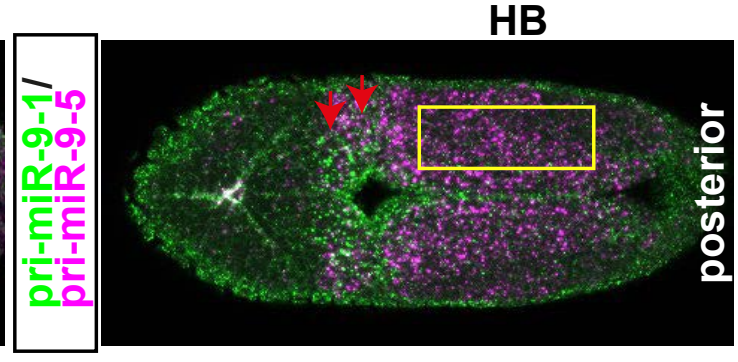

HB
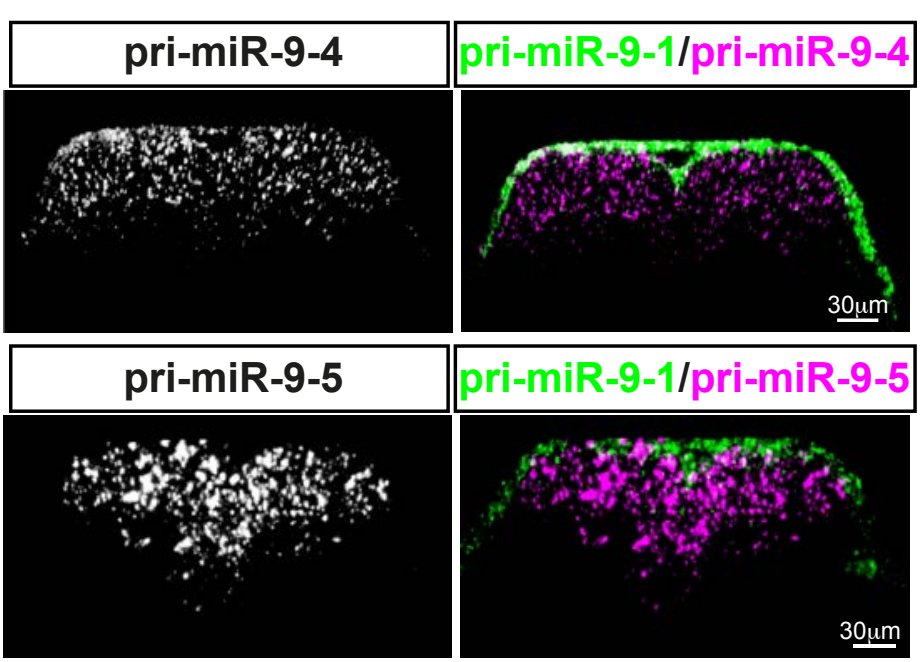

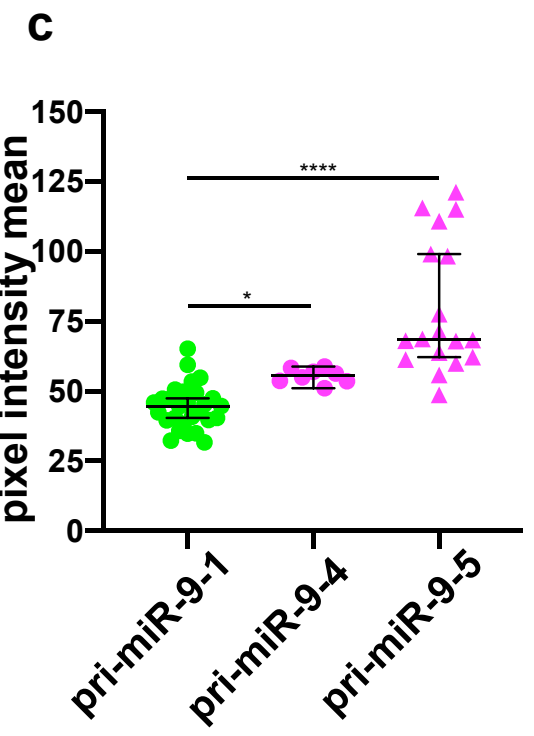

f

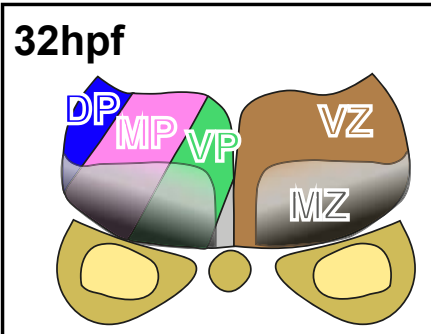

$D P=$ Dorsal Progenitors MP $=$ Medial Progenitors VP= Ventral Progenitors $\mathrm{VZ}=$ Ventricular Zone $M Z=$ Mantle Zone 


\section{Figure 3}

bioRxiv preprint doi: https://doi.org/10.1101/2020.08.03.233890; this version posted August 3, 2020. The copyright holder for this preprint (which was not certified by peer review) is the author/funder, who has granted bioRxiv a license to display the preprint in perpetuity. It is made available under aCC-BY 4.0 International license.

a
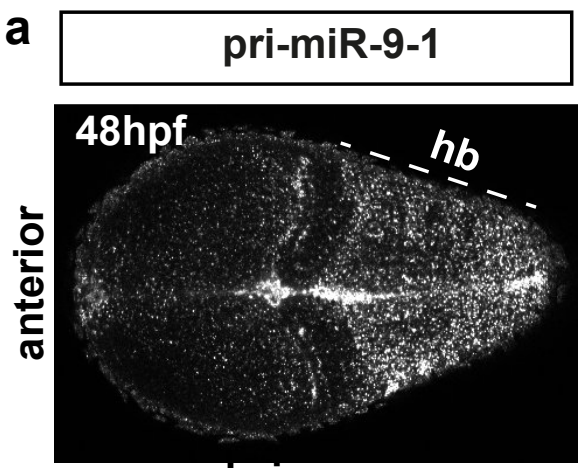

coronal view

b

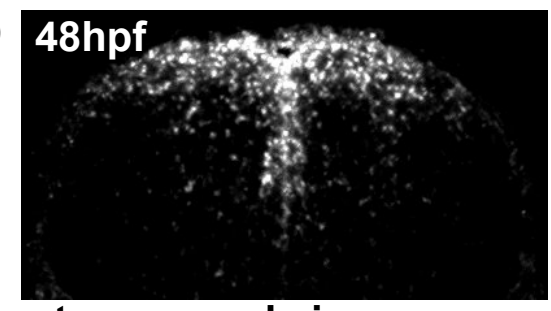

transversal view

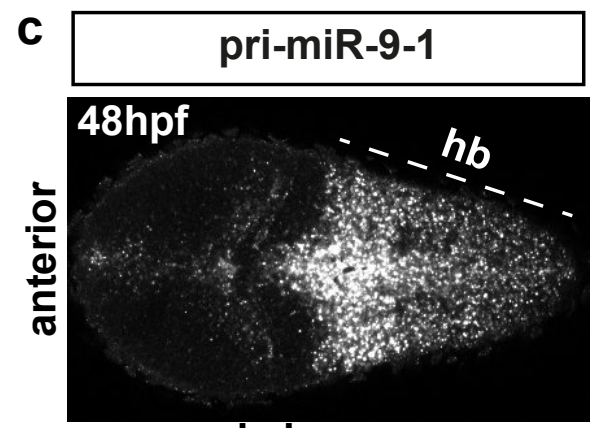

coronal view

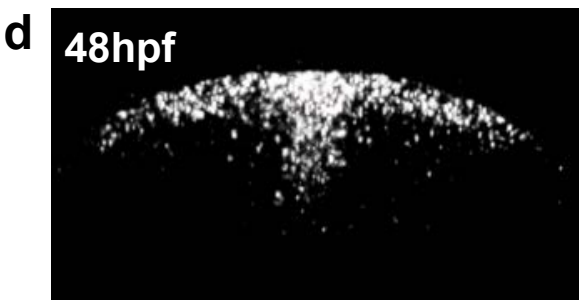

transversal view

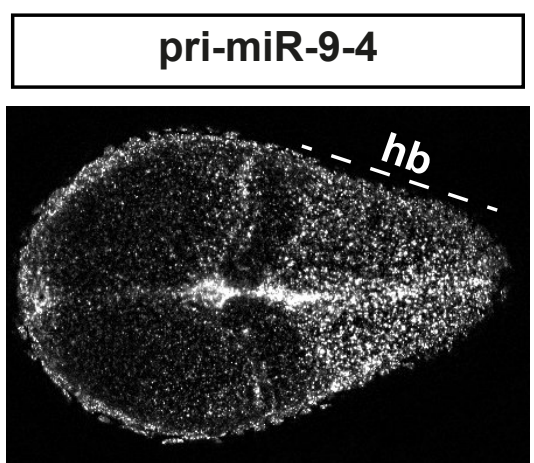

prí-miR-9-1/pri-miR-9-4
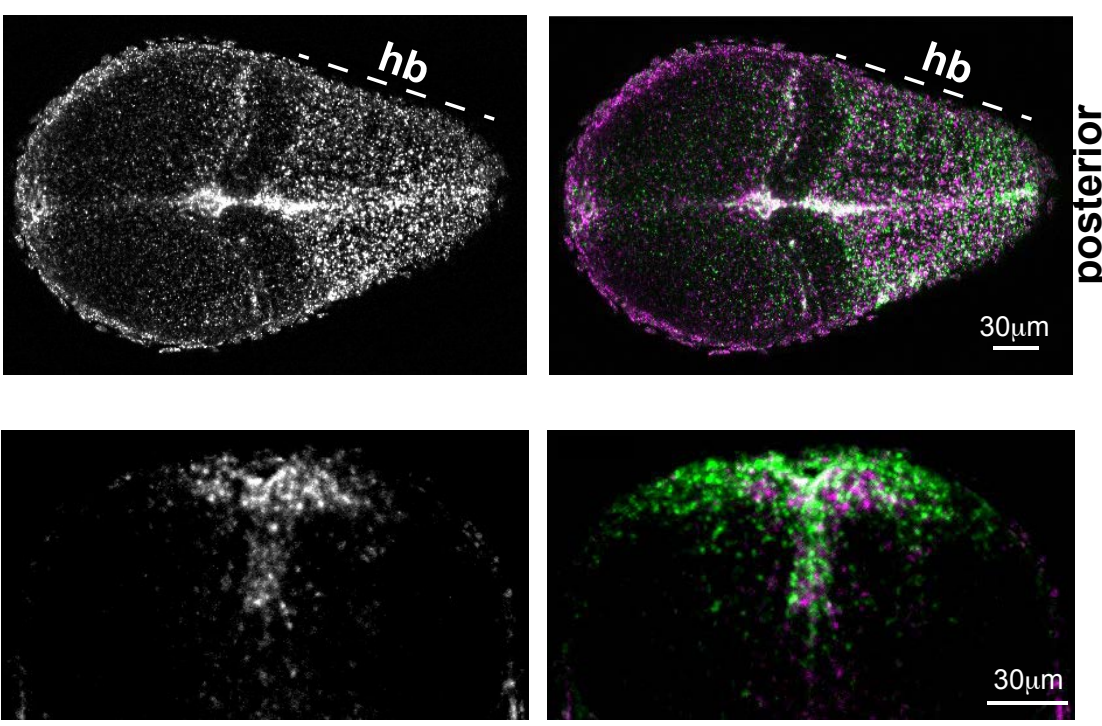

$0 \mu \mathrm{m}$
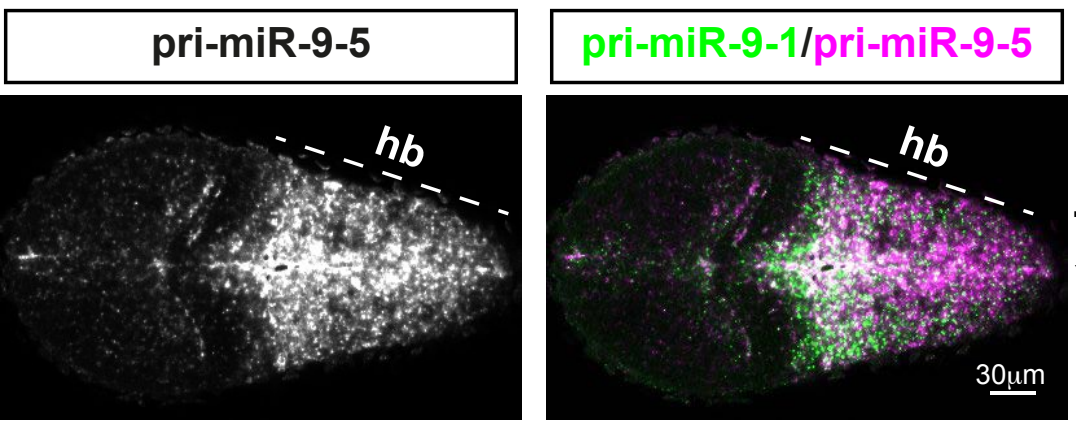

흔
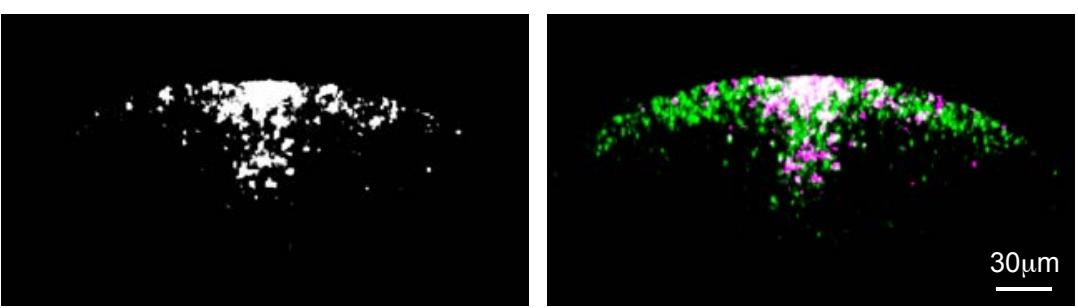

30um

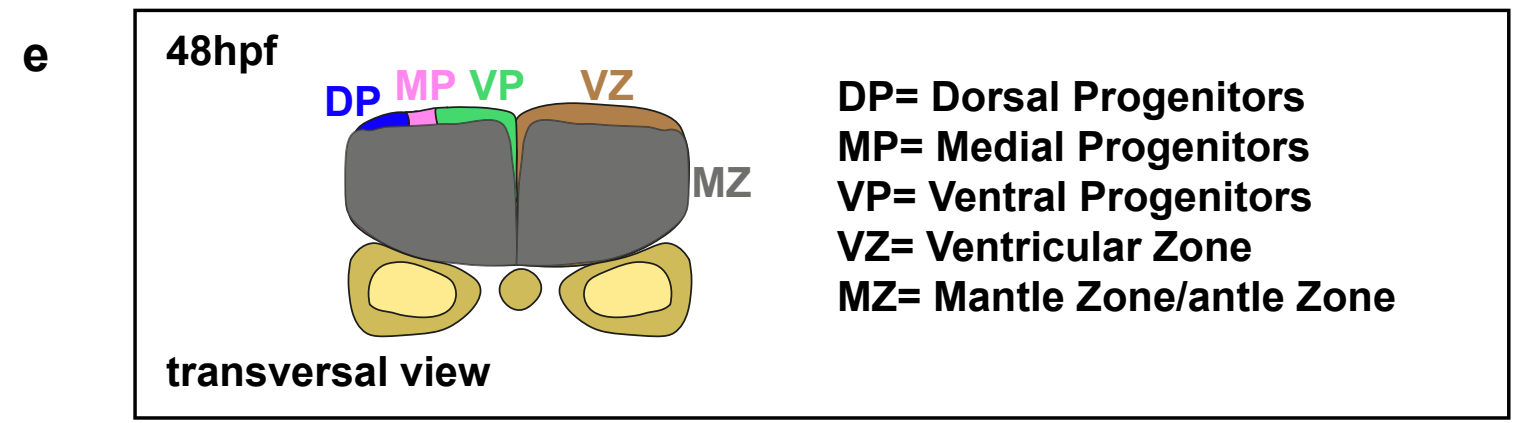




\section{Figure 4}

a

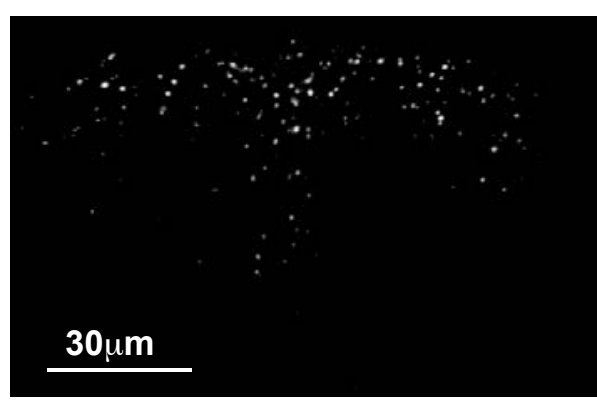

pri-miR-9-1/pri-miR-9-4

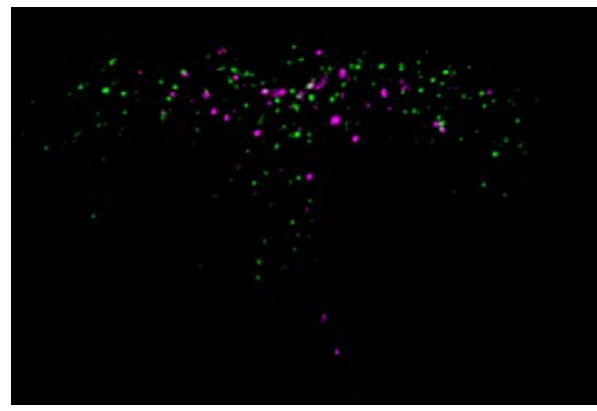

e

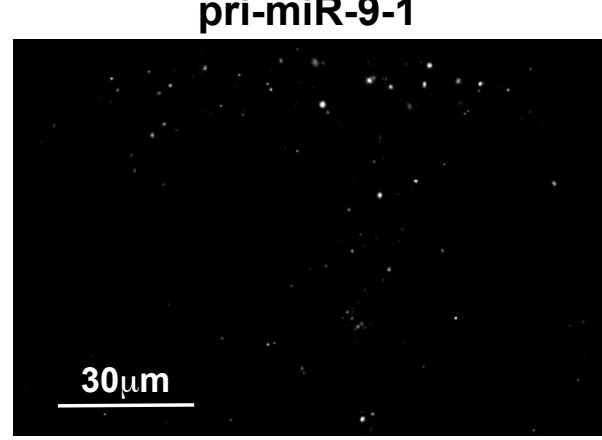

prï-miR-9-1/pri-miR-9-5

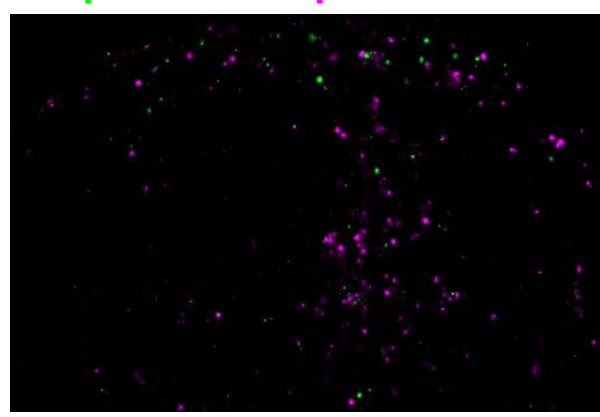

pri-miR-9-1/pri-miR-9-4/BP-TR

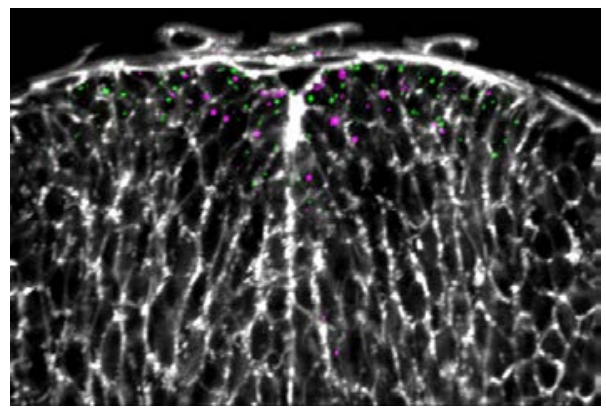

pri-miR-9-5

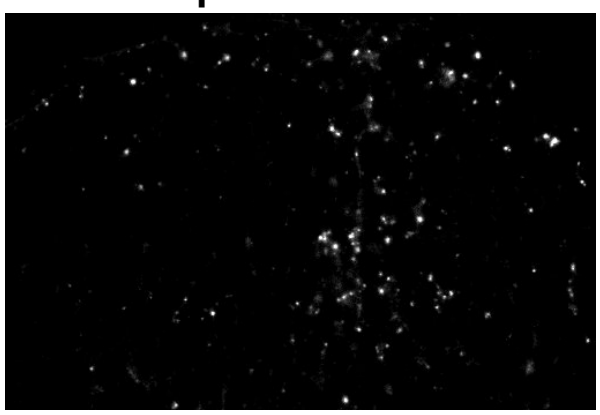

prì-miR-9-1/pri-miR-9-5/BP-TR

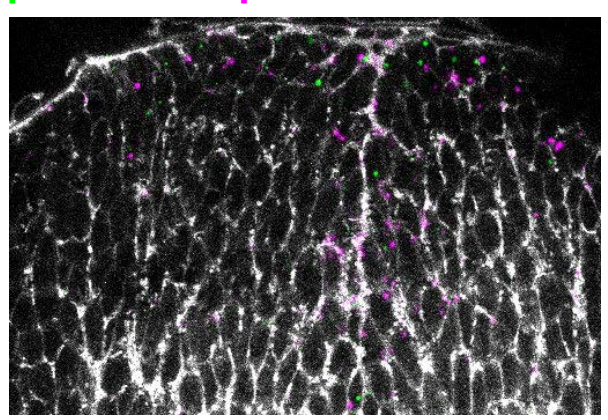

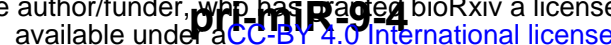
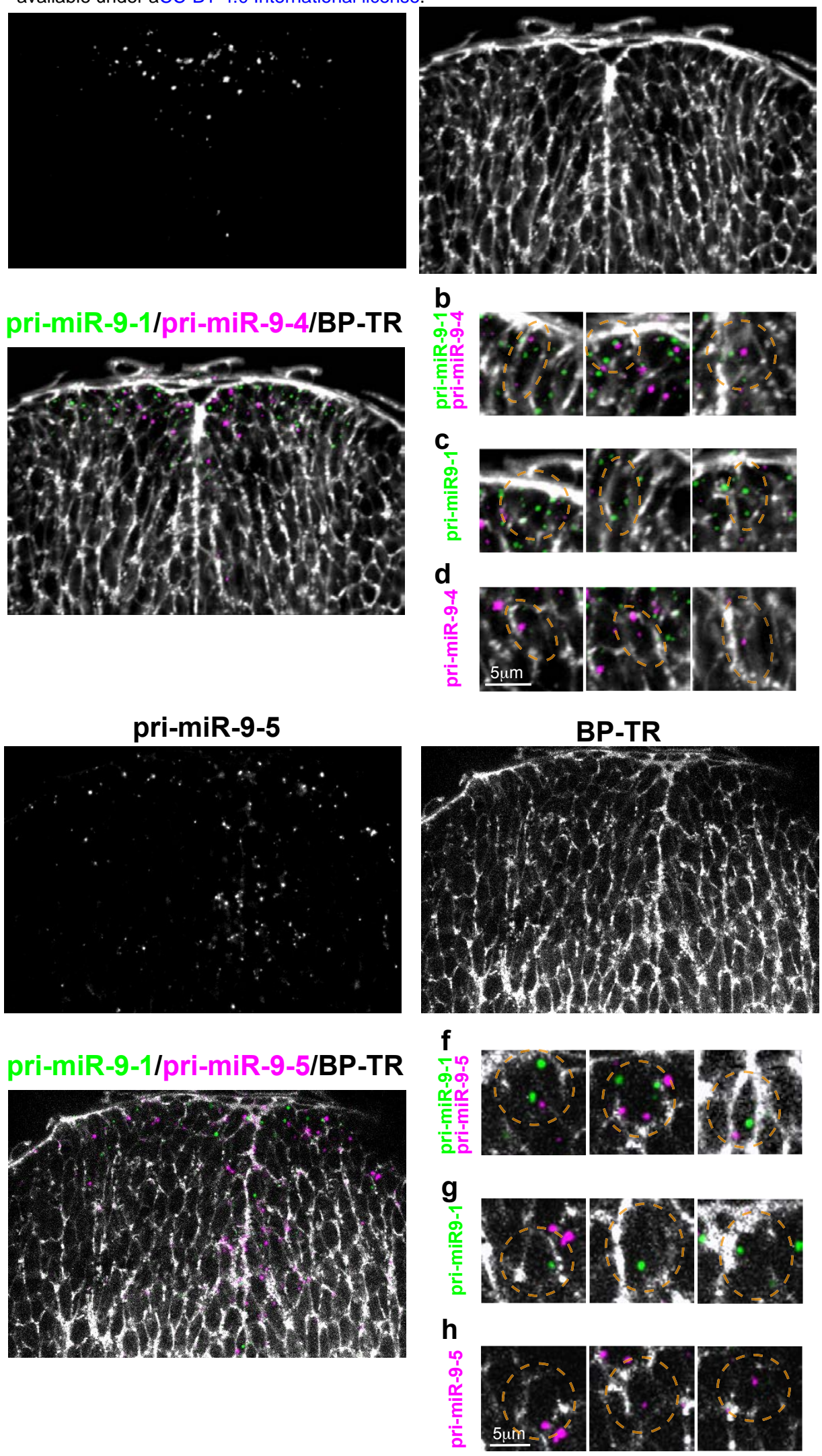


\section{Figure 5}

a | bioRxiv preprint doi: https://doi.org/10.1101/2020.08.03.233890; this versibh posted August 3, 2020. The copyright holder for this preprint which was not certified by peer review) is the author/funder, who has granted biof xiv a license to display the priphigtig perpetuity. It is made
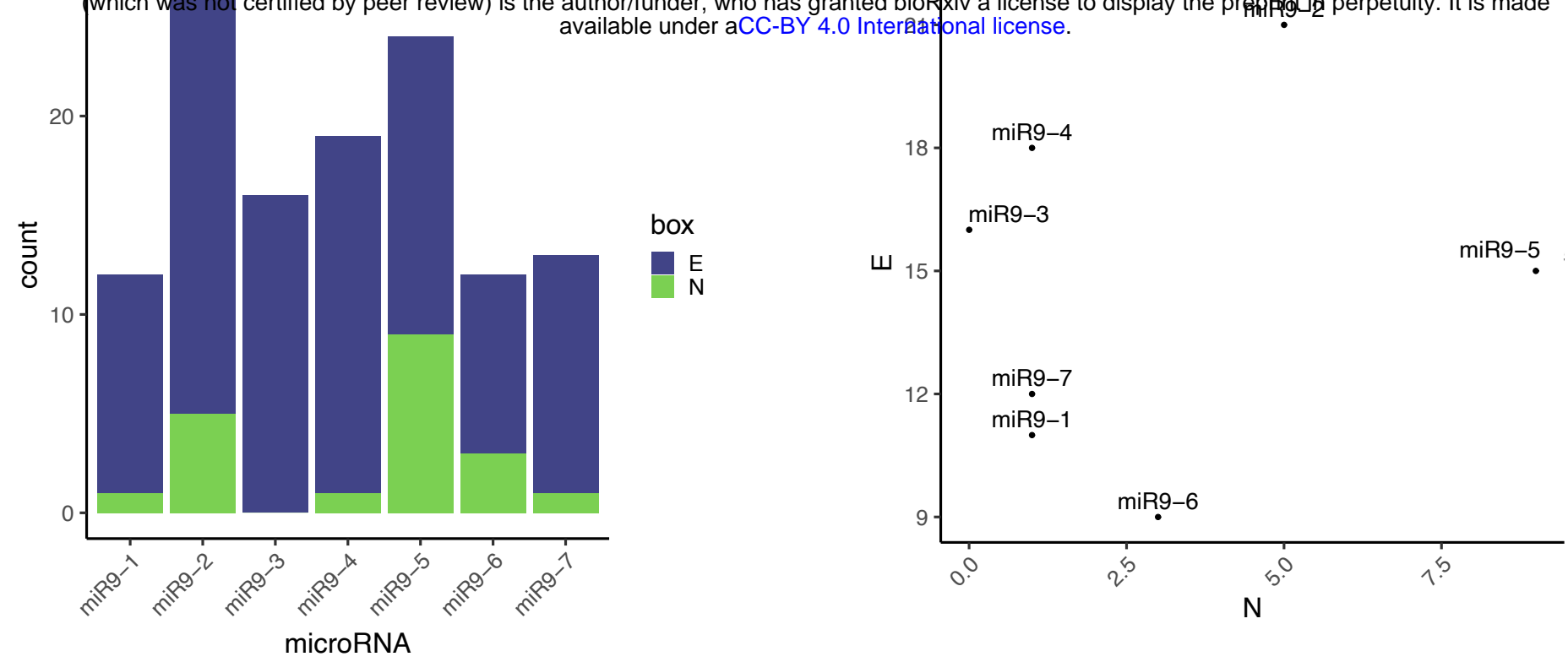

C

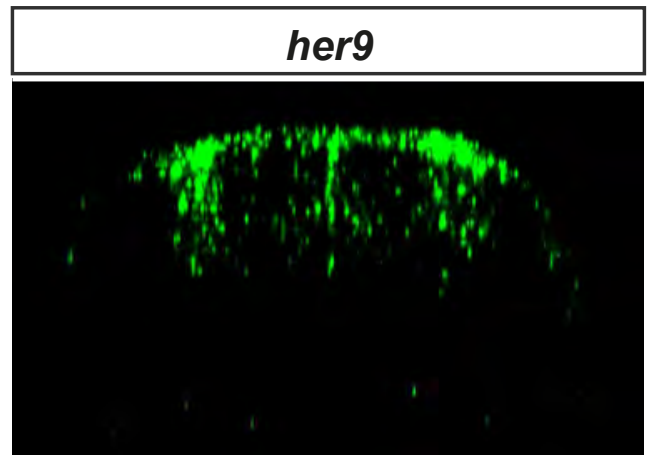

d

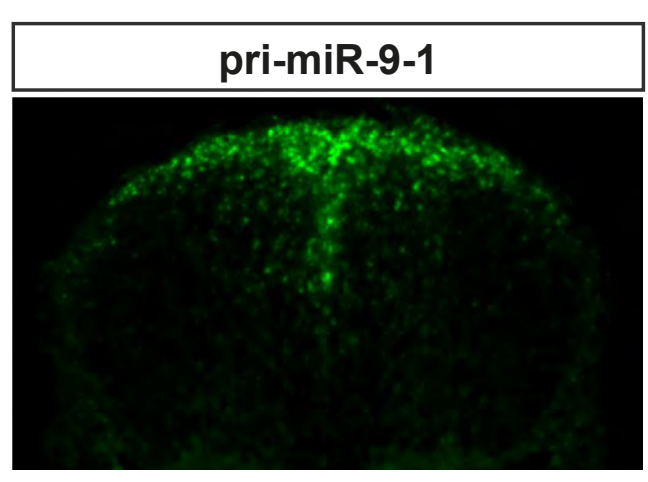

e

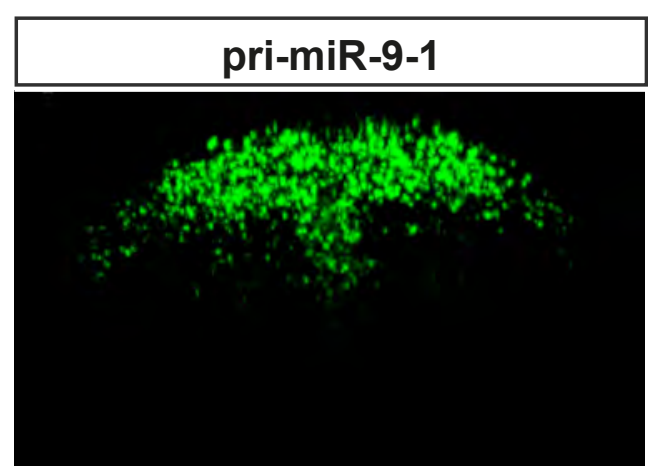

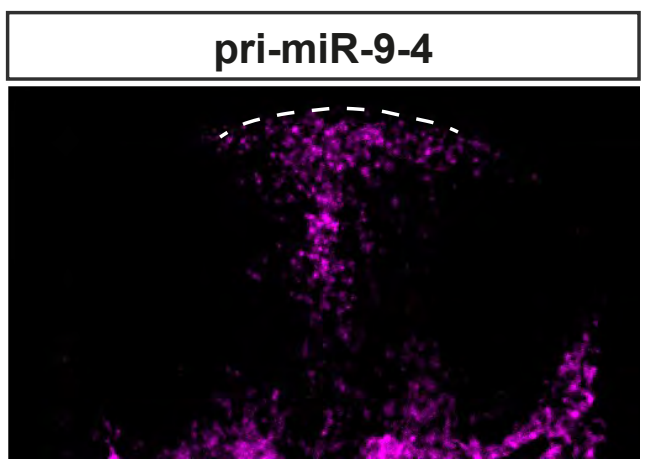
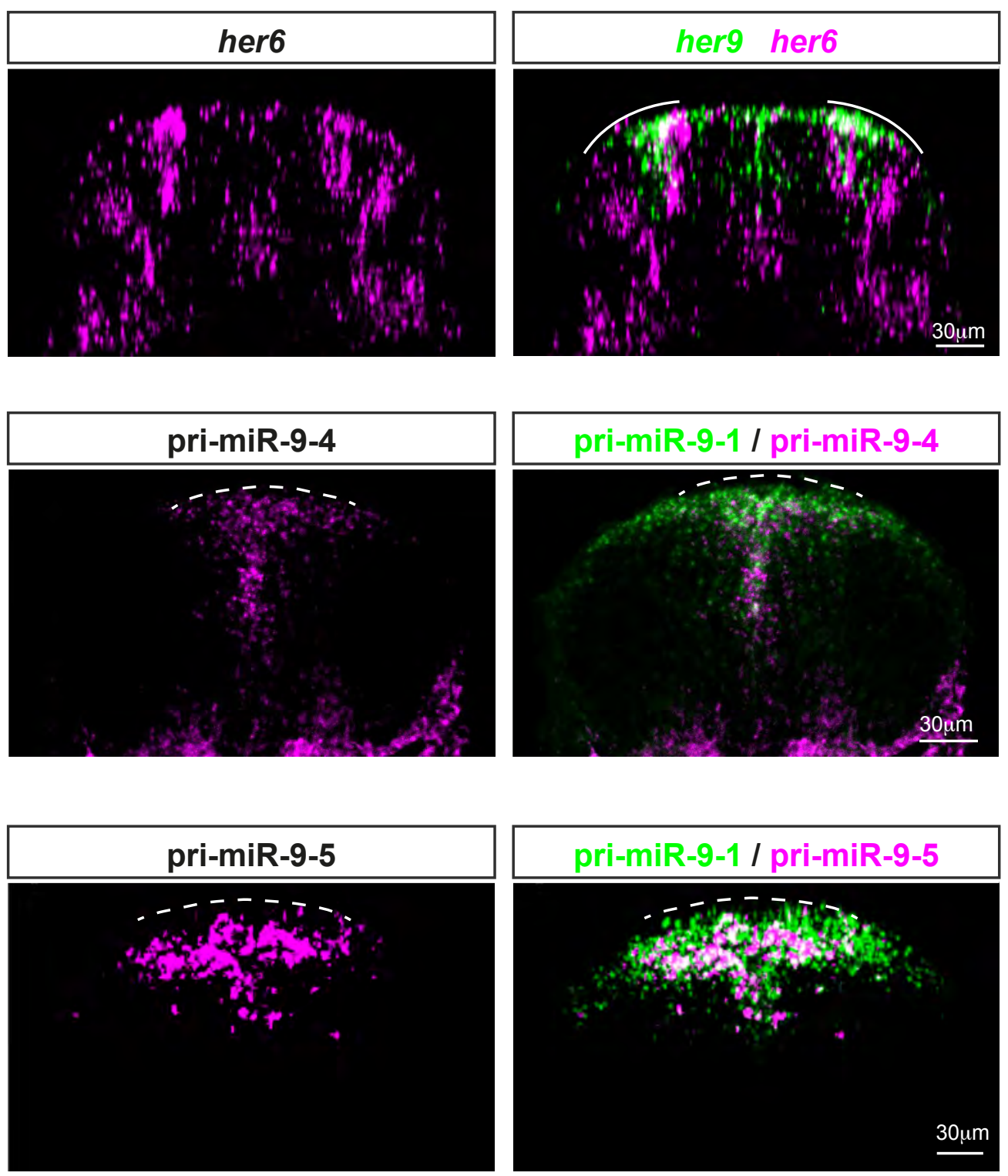